\title{
RESEARCH ON STANDARDIZED ACCESS SCHEME FOR ON - LINE MONITORING DATA OF SUBSTATION
}

\author{
Zhao Dongxu $^{2, a)}$,Cheng Dawei ${ }^{1, b)}$ and Xin Shouye ${ }^{1, c)}$ \\ ${ }^{1}$ The Electric Power Research Institute of Liaoning Province Power Company. \\ ${ }^{2}$ Liaoning Electric Power Co.Ltd. \\ a) zjy1106@sina.com \\ b)chengdawei@dbdky.com \\ c)xinshouye@dbdky.com
}

\begin{abstract}
According to the construction requirements of State Grid Corporation substation condition monitoring system, the on-site monitoring data of the station is connected to the main station system of the state evaluation center, and the evaluation of the primary equipment is completed. However, the early construction of the traditional substation is not carried out according to the national network The relevant monitoring standards of the substation on-line monitoring system can not be accessed into the main station system. Therefore, how to standardize the data in the substation into the state evaluation center has become the focus of a research, This paper first analyzes the framework of the online monitoring system and the idea of the architecture design. In view of the characteristics of the traditional substation construction, this paper puts forward the solution of the traditional substation status monitoring data access to the state evaluation center master station. At present, Provincial power companies within the scope of extensive promotion, through data to prove that the construction of the state evaluation center has important significanc.
\end{abstract}

Key words: Online monitoring system; State evaluation center; CAC; Data standardized access

\section{INTRODUCTION}

In recent years, with the construction of smart grid, substation on-line monitoring system construction has become more and more hot research, but also through the intelligent substation condition monitoring system to complete the construction of a reliable assessment of equipment, and then a device Operation plays a positive and important role. [1-2]. The objective of the construction of the on-line monitoring system of the substation is to provide the reliable analysis of the health status of the primary equipment in order to access the increasingly largescale overhaul equipment maintenance problem, complete the online real-time analysis and diagnose the online monitoring data, and according to the construction of the national network Requirements, the substation within the online monitoring data to be uploaded to the provincial company's state evaluation center power transmission equipment status monitoring master station system, and the State Grid Corporation has issued the relevant standards and norms, but these standards and norms suitable for this year to build the Substation on-line monitoring system access work for the early construction of substation on-line monitoring system is still lack of relevant programs. Therefore, this paper presents the substation on-line monitoring standardized access program, and then realize the on-line monitoring data standard access to the state evaluation center transmission and transformation equipment condition monitoring system, thus realizing the reliable evaluation of the primary equipment [3]. 


\section{Introduction of substation on - line monitoring system}

\subsection{Design ideas}

Substation Equipment Online Monitoring Data Standardized Access to Status Evaluation The master system follows the following design ideas:

(1) Through the understanding of the construction framework of the on-line monitoring system of the transformer equipment, a unified substation equipment architecture is established, the modeling of the on-line monitoring data of the substation equipment and the analysis and understanding of the data are completed, and the technology of the monitoring sensor is mature And the availability of monitoring data for the availability of a device to achieve a scientific distribution.

(2) Substation deployment of a wide range of sensors and other characteristics, resulting in the current substation deployment within the online monitoring system structure are different, so if you want to achieve substation equipment online monitoring system in the national network within the unified deployment and application, we must establish a unified Of the information access specification layer, to achieve the state monitoring data of standardized access.

\section{2 system framework}

In accordance with the State Grid Corporation of substation equipment on-line monitoring system construction requirements, substation equipment status monitoring master system requirements in the State Grid Corporation and the network province two levels of deployment, while in the substation in accordance with the three-tier structure design ideas, namely: Layer, interval layer and station control layer. The process layer is mainly deployed in the monitoring device, the interval is deployed at the station side monitoring unit, station control layer deployment station monitoring unit [4]. Substation equipment status monitoring data in the provincial and national network companies were stored.

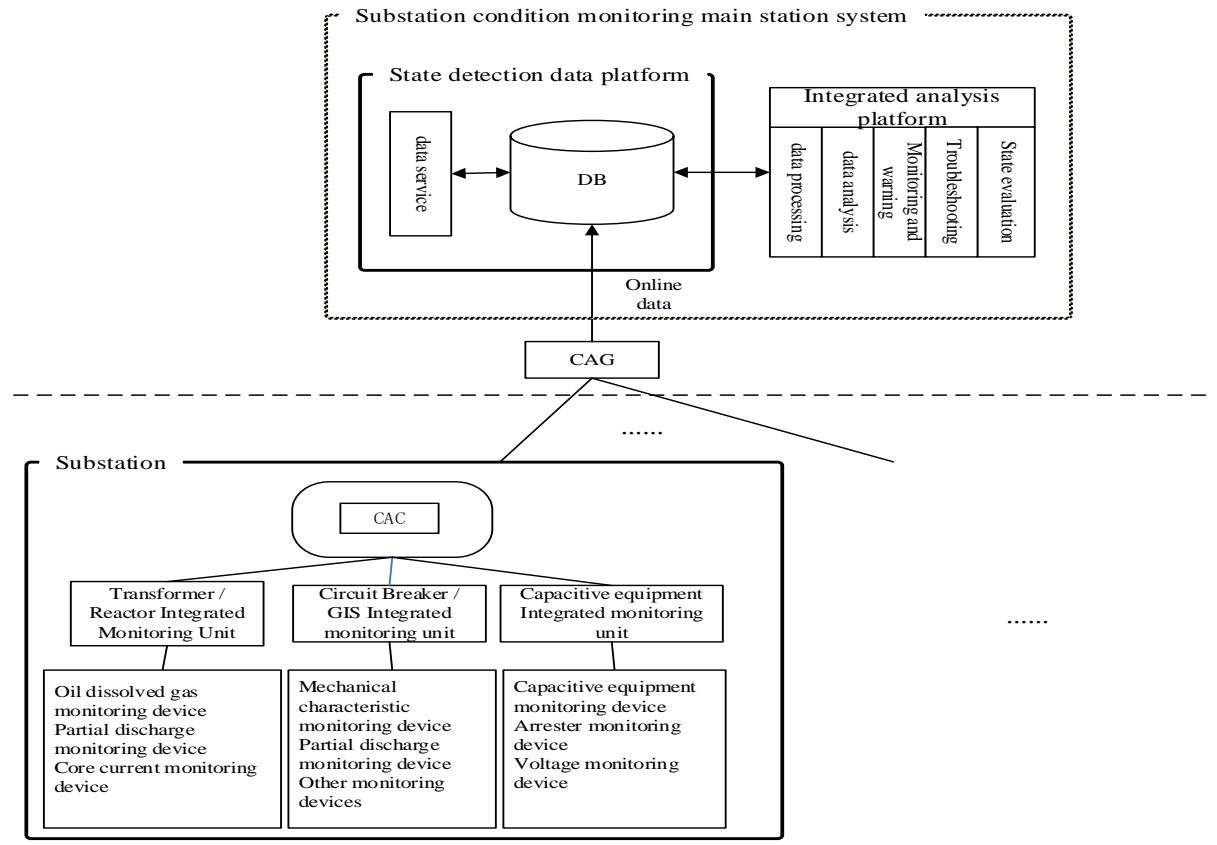

FIGURE 1.Equipment monitoring system for substation equipment

The process layer, the interval layer and the station control layer in the three-layer structure of the station are described as follows: The monitoring device in which the process layer is deployed is divided into monitoring devices that meet IEC 61850 and monitoring devices that do not meet IEC 61850. The main role of the integrated monitoring unit deployment at the interval is to achieve the conversion of monitoring data that does not meet the IEC 61850 monitoring device to meet IEC 61850 monitoring data. The function of the station side monitoring unit 
of the station control layer is to realize the aggregation of the monitoring data in the station and to judge the monitoring data and to complete the monitoring data to the state evaluation center master station system.

In the province of the deployment of power transmission equipment status monitoring system, through the State Grid Corporation of the standard system interface for other types of systems to achieve data sharing to meet the different systems of business data needs. The on-line monitoring system of the substation equipment sends the alarm data to the dispatching master station system at the same time when the alarm data is transmitted to the dispatching, for example, when the monitoring data collected in the station is alarmed.

\subsection{Architecture design}

According to the system construction of the online monitoring system of the national network transmission equipment, a hierarchical architecture system is adopted in the substation, the process layer, the interval layer and the station control layer, the process layer and the interval layer are deployed in the station, between the process layer and the interval layer The communication is done through the manufacturer's private agreement. The communication between the interval layer and the station control layer is completed by IEC 61850 communication. The communication between the station control device and the master station is done through the communication of the I2 interface.

\section{On - line monitoring information access scheme for substation equipment}

Substation on-line monitoring system construction form 1: in the substation deployed within the online monitoring device is isolated, the site of the data can only be the way the country to complete the data collection. As shown below:

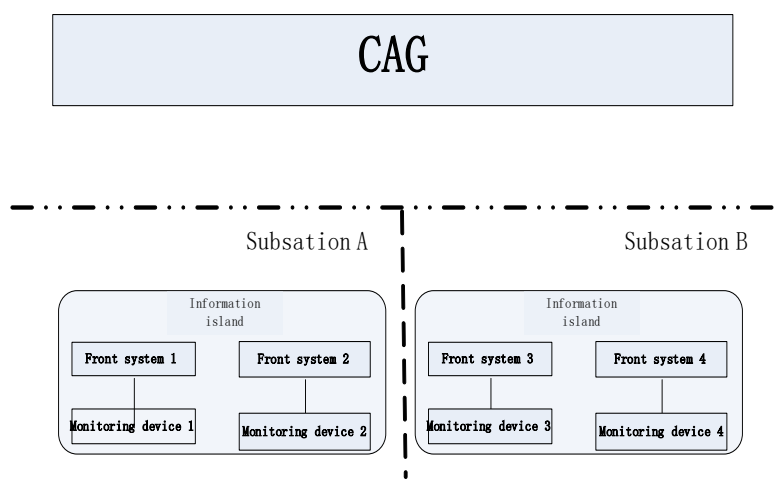

FIGURE 2.Online monitoring system exists in the form of the station

Maintain the normal monitoring of the existing monitoring device, in the station site monitoring unit, the first monitoring device monitoring data through a unified agreement to the station to the end of the monitoring unit, the station side of the monitoring unit to complete the total station monitoring data summary , And the final data through the standard formof transmission to the power transmission equipment status monitoring master station system, where there is only standard standard I2 standard protocol. As shown below. 


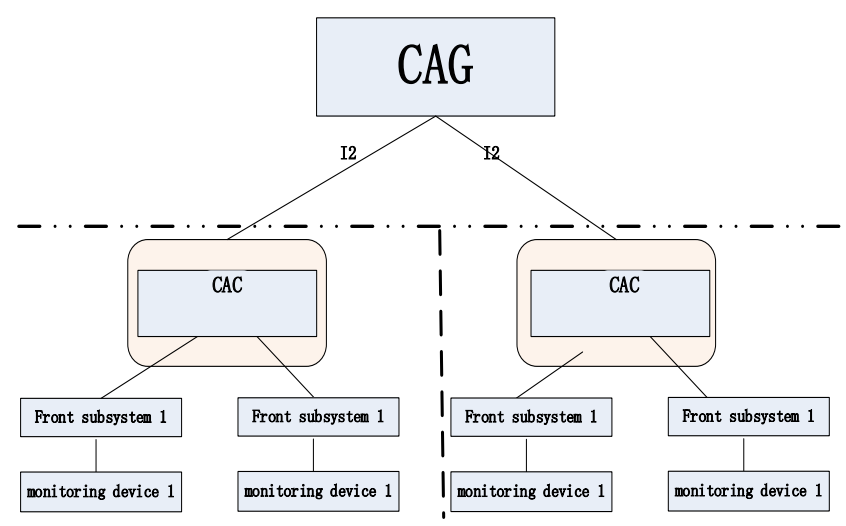

FIGURE 3. I2 standard protocol

Remodeling method 1: In the case of ensuring the operation of the existing monitoring device, the monitoring device monitoring data access to the station control station side of the monitoring unit, and then through the standardization of the I2 communication protocol unified to the power transmission equipment status monitoring main Station system, if the station installed a large number of monitoring devices, unified access to the station side of the monitoring unit will be the end of the monitoring unit load a lot of inconvenience. As shown below:

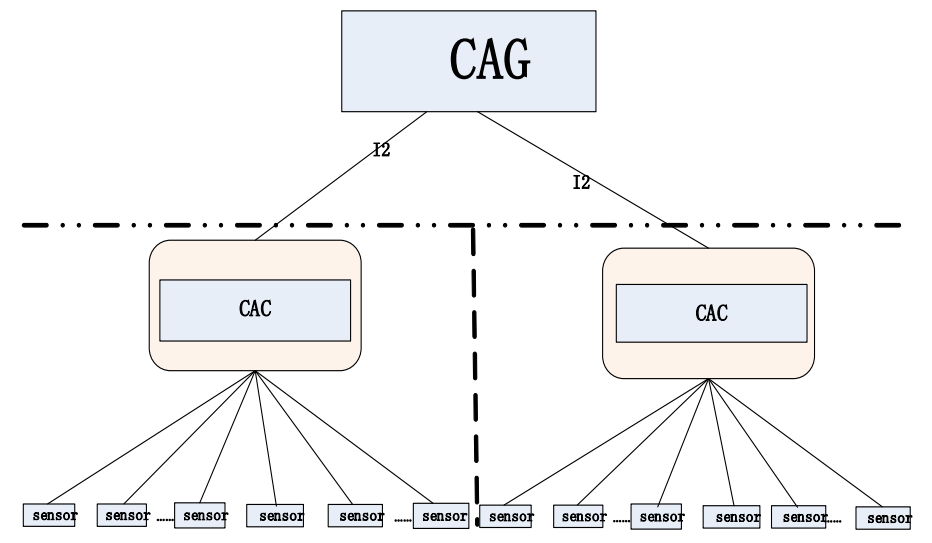

FIGURE 4.Deployment station monitoring unit transformation program

\section{CONCLUSION}

The construction of on-line monitoring system for substation equipment has become an important direction for the intelligent construction of State Grid Corporation. In recent years, the State Grid Corporation has issued relevant guidelines and specifications for the construction of on-line monitoring system for substation equipment. Support, but the traditional substation monitoring data access to the state evaluation center of the master station system is still missing the relevant detailed solution, so this article for the current substation within the online monitoring system construction ideas, put forward a standardized access Method, the purpose is to substation on-line monitoring data standardized access to provide more options.

\section{REFERENCES}

1. State Grid Corporation,Q / GDW383-2009, 《Technical Guidelines for Intelligent Substation》, Beijing: State Grid Corporation Office, 2009-12-28.

2. State Grid Corporation, Q / GDWZ410-2010, 《Guidance on intelligent technology for high - voltage equipment》, Beijing: State Grid Corporation Office, 2010-02-10.

3. State Grid Corporation, Q/GDW 534-2010, 《Technical Guidance for On - line Monitoring System for Transformer Equipment》 , Beijing: State Grid Corporation Office, 2011-04-28. 
4. State Grid Corporation, 《Summary Design of Condition Monitoring System for Power Transmission and Transformation Equipment》, Beijing: State Grid Corporation Office, 2010-06-04.

5. State Grid Corporation, Q/GDW 749-2012 《Technical specification for state access controller of substation equipment》, Beijing: State Grid Corporation Office, 2012-06-21.

6. State Grid Corporation, Q/GDW 739-2011, 《Specification for on - line monitoring I1 interface network communication for substation equipment》 , Beijing: State Grid Corporation Office, 2012-08-02

7. State Grid Corporation, Q/GDW 740-2011, 《Specification for on - line monitoring of I2 interface network for substation equipment》, Beijing: State Grid Corporation Office, 2012-08-02.

8. State Grid Corporation, 《Technical specification for integrated monitoring unit of on - line monitoring system for substation equipment》 